\title{
Non-classical metatheory for non-classical logics
}

\author{
Andrew Bacon*
}

January 10, 2012

Non-classical logics have been applied to a number of problems in philosophy. Notable applicatons include the Sorites and the Liar paradoxes, although the list extends far beyond these. It is usual in such applications to specify the logic in question by the means of a classical metatheory: a classical description of a class of models, typically involving multiple truth values, which characterises the logic we are interested in.

A number of authors have found this discrepancy between the logic espoused and the logic used to reason about the logic espoused to be an embarrassment. For example, in his influential criticism of non-classical approaches to the Sorites paradox, Timothy Williamson writes:

There is a problem. The many-valued semantics invalidates classical logic. Thus if the metalanguage is to be given a many-valued semantics, classical reasoning is not unrestrictedly valid in the metalanguage. [23], p128

Hartry Field, in a similar context, writes:

If we are to take seriously the idea that vagueness or indeterminacy is a quite widespread phenomenon, then we should consider the possibility that the language in which we discuss the semantics of vague and indeterminate language will itself be vague or indeterminate; and then if classical logic can't be used with vague or indeterminate language, we won't even be able to use classical logic in metatheoretic reasoning about the logic of vague or indeterminate language. [3], p10

The phenomenon of higher order vagueness, however, is just a case in point; it has been noted, concerning non-classical approaches to the semantic paradoxes, that a classical semantic theory provides the resources to form a revenge liar sentence (see, for example, Field [4] and Leitgeb [11].) Similarly, Gödel and Kreisel have shown certain metatheoretic results about intuitionistic logic can only be proved using non-constructive reasoning (see Kreisel [9].) The general moral seems to be that the very phenomena responsible for non-classicality occur just as frequently in the field of semantics as elsewhere.

*I would like to thank Cian Dorr, Timothy Williamson, Bernhard Salow and two anonymous reviewers for their many helpful comments on this paper. 
In [4] Field proposes that we separate the project of giving a model theory for a logical language from the project of giving it a semantics. A model theory is supposed to characterise what follows logically from what. Semantics, on the other hand, is the study of the intended meanings of the language's words, and is only concerned with the language's intended model. The primary objections to classical metalogic discussed in the last paragraph stem from non-classicality arising in the semantic theory, and not in the model theory. Field argues that we can characterise the extension of his preferred non-classical consequence relation in a classical model theory, provided that one keeps in mind that this model theory does not contain the intended model. ${ }^{1}$

As far as semantics is concerned, Field's non-classical object theory contains certain semantic notions such as a truth predicate or a satisfaction relation. However one might object that this is far from enough to do any serious semantics. For instance, one does not have the resources to theorise about the intended interpretation at the subsentential level: to reason about the semantic values of predicates, predicate modifiers, quantifiers, and so on, one must appeal to resources that go beyond satisfaction and truth.

Field's approach notwithstanding it seems that it ought to be possible to formulate the metatheory of a non-classical logic non-classically. This project has been gestured at by Tye [20] and, more recently, by Leitgeb [11] in the context of Field's theory of truth. However, except in the case of intuitionistic logic, which is too strong to deal with the applications of interest such as the liar and the Sorites paradox, little has been done to make plausible the idea that a non-classical logic can formulate substantial metatheoretic results about itself. The purpose of this paper is to make this claim seem plausible for a very simple language - the propositional calculus - for a large class of weak non-classical logics. The class includes fuzzy logics such Łukasiewicz, Gödel and product logic [7], BCK [6], intuitionistic logics, quantum logic [8], among others. The list does not include logics which do not allow you to infer $\phi \rightarrow \psi$ from $\psi$ or logics without a reasonable conditional. ${ }^{2}$ The logics under consideration also contain rules that one would normally take for granted in a classical setting, such as the 'rule of proof': either you can infer $\phi$ from $\Gamma$ or you can't.

While the current paper is restricted to propositional languages, it is natural to think that some variation on these ideas may survive in the extension to the predicate calculus. Perhaps this is true for at least some applications of

\footnotetext{
${ }^{1}$ There is a puzzle for Field in explaining why and how his models end up validating the correct logic. Welch shows in [21] that the logic of Field's model theory is highly non-recursive. Given Field's instrumentalism about this model theory and the impossibility of surveying everything it validates, we are left with no other way to evaluate its claim to correctness. Perhaps a better interpretation of Field's construction would be as a consistency proof of some suitably chosen recursive subsystem. Another possible interpretation that would not require choosing a particular subsystem would be to treat the truth values in Field's models as credential states in a non-standard representation of rational degrees of belief. Insofar as logic is just whatever plays a certain normative role with respect to our beliefs, this interpretation allows us to formulate an argument that his logic is correct. I leave the interpretive issues to one side for now; the solution presented here is available to Field's theory as much as it is elsewhere.

${ }^{2}$ I take it that the law $\phi \rightarrow \phi$ and modus ponens must hold for any reasonable conditional.
} 
interest, however it is worth mentioning in this regard the results of McCarty [12] to the effect that intuitionistic predicate logic is provably incomplete, within intuitionistic metamathematics, with respect to models of broadly the same kind considered here. ${ }^{3}$

In $\S 1$ I sketch in outline the approach. Classical bivalent model theory, in the style of Tarski, can be carried out in a non-classical metatheory with a suitable amount of set theory. It is explained how it is possible, even in a bivalent model theory, to invalidate classical laws provided that matters of set membership needn't abide by classical logic.

In $\S 2$ a bivalent model theory for the propositional calculus is presented in which logical truth can be defined. It is shown that for a large class of logics, $L$, if the logic of the metatheory is $L$, then every $L$ theorem will be classified a logical truth according to the metatheoretic definition.

In the final sections some puzzling features concerning non-classicality in statements about validity are addressed. As noted, classical logic holds for all provability statements in the logics we consider. However it is shown that validity statements need not be classical. I draw and defend one surprising conclusion for the application of non-classical logic to vagueness: no axiomatic, and therefore precise, system can be determinately sound and complete.

\section{Taking model theory seriously}

Say that a class of models is 'faithful' just in case (i) each model represents a possible way of interpreting the language in question, and (ii) every possible way of interpreting the language is represented by some model. In order to understand this, one needs to have some antecedent understanding of what it means to interpret a language. While detractors may deny that there is any clear notion in the ballpark, there is at least one clear cut difference between a faithful model theory and a Field-style instrumentalist conception of model theory: a faithful model theory must represent the intended intperpretation (by requirement (ii).) The strategy for this paper is to demonstrate that many non-classical logics can be given a faithful model theory by construing relatively standard definitions of truth-in-a-model and validity inside a non-classical set theory.

The project of giving a logic a faithful model theory dates back to Tarski's influential account of logical consequence [19] and has the benefit of allowing straightforward explanations of the normative role, necessity and formality of logic that Field-style instrumentalism about model theory lacks.

It is not at all clear, however, whether the modern set theoretic model theory for first order logic is faithful. While it is arguably 'semi-faithful' in the sense that it satisfies condition (i), it is often pointed out that it is not fully faith-

\footnotetext{
${ }^{3}$ Although it should noted that these results are distinctly intuitionistic and rely on mathematical principles that contradict classical mathematics - McCarty relies on this fact, by showing that classical arithmetic is semantically inconsistent in the sense that it has no Tarskian models.
} 
ful because it fails to represent the intended interpretation and other possible interpretations of a first order language which are too large to form a set.

I think there are two points that ought to be made at this juncture. Firstly, the choice to formulate one's model theory in terms of sets is a rather superficial one. The metatheory of Tarski's original definition of logical consequence, for example, wasn't ZFC but a type theory in which the existence of an intended model follows immediately from an instance of universal instantiation (see [19] and [15].) More recently definitions of logical truth and consequence for first and second order logic have been given using a metalanguage containing plural or second order quantifiers ([17], [18].)

Secondly, while the classical set-theoretic model theory for first order logic is not faithful, we can in this instance prove that the restriction to set sized models does not affect the extension of the logical consequence relation. ${ }^{4}$ This argument, due to Kreisel [10], relies on an antecedent conception of validity perhaps something closer to Tarski's initial conception - and appeals to property (i) of the set-theoretic models. In the non-classical case, however, the issue for the instrumental algebraic model theory is precisely that (i) fails: no classically described algebraic model represents a possible interpretation of the language. Those models are just mathematical objects which happen to be useful for showing when a theory is consistent, or that something doesn't follow from something else.

It's unclear whether it's possible to justify the use of a particular instrumentalist algebraic model theory unless one has some independent grasp of what is valid to compare it too. If we had an independent definition of validity it may be possible to run something analogous to the Kreiselian justification of the set theoretic model theory for the classical predicate calculus. However the logic validated by an algebraic model theory can be highly non-recursive; in these cases our only access to the logic is through the supposedly instrumental model theory. This is true with Field's algebraic model theory for example - our grasp of validity in this case is dependent on his model construction. Another approach would be to specify a logic independently by an axiomatisation. We could justify the soundness of an instrumental model theory by proving its completeness for the axiomatic logic, and then we could argue that the axiomatic logic was intuitively sound. But the axiomatic way of specifying a logic leaves no guarantee that the logic contains all the principles it intuitively ought to have. Of course, it is debatable whether, say, an axiomatic paracomplete logic is complete in the absolute sense, because it is debatable whether the law of excluded middle is a valid sentence. The kind of incompleteness I am worried about does not turn on debatable principles: sometimes it is a non-trivial matter whether

\footnotetext{
${ }^{4}$ Suppose that $\Gamma \vdash \phi$ where $\vdash$ represents provability in standard axiomatisation of first order logic. Since the axioms and rules of inference are evidently logically true and logically truth preserving respectively it follows that $\phi$ is a logical consequence of $\Gamma$. That is to say any possible interpretation of $\mathcal{L}$ making $\Gamma$ true makes $\phi$ true. Since any set sized model is a possible interpretation of $\mathcal{L}$ it follows that $\Gamma \models \phi$. Thus logical consequence is sandwiched between $\vdash$ and $\models$; i.e. it contains $\vdash$ and is contained in $\models$. However, since $\vdash=\models$ by Gödel's completeness theorem, it follows that logical consequence is just the same as $\models$.
} 
something which is uncontroversially valid is provable in a given system. For example the early axiomatisations of free and quantified modal logic did not prove the principle $\forall x \forall y \phi \rightarrow \forall y \forall x \phi$ [5]. Even though there was no consensus at that time about which exact modal logic was the right one, substantial questions could still be raised about the completeness of an axiomatic system.

\subsection{Vague sets}

Tarski provided for classical logic an explicit definition of validity in a faithful model theory. ${ }^{5}$ Classical logic therefore meets the challenge raised in the previous section; one can formulate an intuitively correct definition of validity to which classical logic is provably sound and complete by its own lights. In order to meet the challenge in a non-classical setting, I propose that we investigate the prospects of a faithful model theory for the non-classical logic. One requirement a faithful model theory must meet is to be able to describe the intended model. However, since we are taking the considerations of higher order vagueness, revenge, and so on, to show that one cannot describe the intended model classically, a classical metatheory would be ill equipped to formulate a faithful model theory. Let us now elaborate on this point.

As a metalanguage in which to formulate a faithful model theory, classical set theory has two limitations. We have noted already that it is limited to interpretations in which the domain and the interpretation of the predicates are set sized. However, once one has admitted the existence of predicates that invalidate the principles of classical logic, classical set theory may fail to provide interpretations for these predicates as well.

A paracomplete logician working in a standard axiomatisation of set theory must assume a restricted form of the law of excluded middle, $x \in y \vee \neg x \in y$, as a non-logical axiom, in order to recover the full strength of classical set theory. In so restricting our theory of sets we restrict the possible ways of interpreting a predicate to crisp, classical sets. We therefore will miss out on the intended interpretation, not just because the intended interpretation of some predicates are too large to form a set, but because the intended interpretation of 'bald', i.e. the set of bald things, is not a classical set. Such a set is not in the range of the quantifiers of a classical set theory, since we have stipulated that every set in its remit are such that statements about its members obey the law of excluded middle. If we relax the axiom $x \in y \vee \neg x \in y$, and permit vague and indeterminately specified sets, however, it is no longer clear that every classical theorem will be true on every interpretation constructed from such indeterminate sets.

To make this more concrete, let us suppose we are working in ZFCU (ZFC with urelemente) with an open ended formulation of the axiom of separation:

$$
\forall x \exists y \forall z(z \in y \leftrightarrow(\phi \wedge z \in x))
$$

\footnotetext{
${ }^{5}$ I am here referring to Tarski's original definition of consequence, [19], and not the later set theoretic version.
} 
Here $x$ and $y$ are not free in $\phi$. To say that this schema is understood 'open endedly' is to say that we should continue to accept its instances no matter how we extend the vocabulary of our language (see [14].) Unlike the standard formulation of separation, we should accept (1) even when $\phi$ is not stated in the language of set theory. For second order logic one can make a similar point about the comprehension schema: in both cases the intention is to force there to be as many collections of objects as the logic permits. For otherwise one could augment the language with a logically coherent predicate that is not determinately coextensive with any set or collection.

Having relaxed this restriction on separation it is natural to ask if we can infer the existence of more sets than we otherwise could have. The answer to this question will depend on the background logic. For a thorough going classical logician the answer is 'no'. For example, the instance of (1) obtained by substituting $\phi$ for the predicate ' $x$ is small' (as applied to natural numbers) will allow us to directly infer the existence of the set of small natural numbers. However, under the assumption that 100000 isn't small, and the assumption that if $x$ is small and $y \leq x$ then $y$ is small, the classical logician can already infer this fact without the extended separation scheme. She can already prove, by classical logic, that for some $N$, every number less then $N$ is small and every number greater than or equal isn't. On the other hand, from the restricted separation theorem we can prove $\forall n \exists x \forall y(y \in x \leftrightarrow y<n)$, since $<$ is definable from $\in$. So in particular you can prove $\exists x \forall y(y \in x \leftrightarrow y<N)$, and therefore $\exists x \forall y(y \in x \leftrightarrow y$ is small $)$.

For someone who denies that vague predicates obey the laws of classical logic this reasoning cannot always be carried out. ${ }^{6}$ Therefore in some cases the open ended separation schema is a genuine strengthening of the version restricted to set theoretic vocabulary.

Let's now look at a toy example. These ideas will be developed more thoroughly in the next section. The idea is to give a completely standard semantics for our language with the proviso that we relax the assumption that the sets we are working with are all classical sets. With this in place we can see how, even on standard classical semantics, classical laws can fail.

Let's take the propositional calculus, with a countable set of propositional letters $\left\{P_{n} \mid n \in \mathbb{N}\right\}$. A model is, as usual, a function $v: \mathbb{N} \rightarrow 2$ - or, a set of ordered pairs. $v$ may or may not be a vague set. I shall assume, deferring a rigorous account until later, that we can understand what it means for $v$ to satisfy a formula even when $v$ is a vague set. We shall talk about a sentence, $\phi$, of the propositional calculus, being valid - which is to be short for the assertion that every model, $v$, satisfies $\phi$.

\footnotetext{
${ }^{6}$ For example any classical model of set theory is also a degenerate example of a Lukasiewicz model, in which every sentence of set theory receives value 1 or 0 . It is easy to expand this to model the language with 'small' in a way that $\exists x \forall y(y \in x \leftrightarrow y$ is small $)$ has an intermediate value by letting ' $x$ is small' receive intermediate values. On the other hand, the reasoning used to prove this formula in the classical case is also intuitionistically acceptable. We must decide on a case by case basis whether a given set will exist in a logic with the restricted separation scheme.
} 
Suppose that, in a given context, it is vague whether $k$ is small. By open ended separation on $\mathbb{N} \times 2$ we get a function $v$ :

$$
v\left(P_{i}\right):= \begin{cases}1 & \text { if } i \text { is small } \\ 0 & \text { otherwise }\end{cases}
$$

$v$ here is 'bivalent' in the sense that the codomain of $v$ consists of two truth values: $\{0,1\} .^{7}$

We can now see how on certain paracomplete accounts of vagueness this model theory fails to validate excluded middle, despite being bivalent. If excluded middle were valid, then the instance $\left(P_{k} \vee \neg P_{k}\right)$ would be satisfied by every model. In particular $\left(P_{k} \vee \neg P_{k}\right)$ would be satisfied by $v$, which happens precisely if $k$ is small, or $k$ is not small. Since, we may assume, this latter claim is at best vague, it is at best vague whether $\left(P_{k} \vee \neg P_{k}\right)$ is true on $v$ and thus it is at best vague whether excluded middle is true in every such model.

This informal reasoning could be made precise in a suitable paracomplete logic such as Łukasiewicz logic. However this example is deficient in several respects. For a start not every non-classical logic is paracomplete so the impact of this example is rather limited. More importantly, though, we don't have a general argument that the classical laws that fail in our logic, whatever they may be, will always be laws that fail to be validated in the corresponding nonclassical model theory. To ensure this one needs to make sure there are enough sets, and hence enough models, to invalidate any possible law that is not logically valid: that there are as many collections over the domain as the logic in question permits. In this section we have shown this conception of set or collection can be fixed by understanding the separation schema open endedly (similar points apply to the second order quantifiers and the comprehension schema.) In the next section we show how to interpret second order quantification or quantification over sets in a way that ensures the separation/comprehension schema hold open endedly.

\section{A non-classical metatheory for the proposi- tional calculus}

In our discussion so far I have suggested that models constructed from nonclassical sets can invalidate classical laws. But this is clearly not enough to deflect the objection raised against classical metatheories for non-classical logics: that it validates a different logic in the object-language than the logic of the metalanguage. What would a satisfactory response to this look like? In his discussion of degree-theoretic logics for vagueness, Williamson suggests the following constraint:

\footnotetext{
${ }^{7}$ To say that $D$ is the codomain of a function $f$ is to say that $\forall x(\exists y f(y)=x \rightarrow x \in D)$. In the logics considered the codomain of $v$ being $\{0,1\}$ does not entail that $v(x)=1 \vee v(x)=0$ for any $x$.
} 
On the degree theoretic account, what is an appropriate logic for a vague language? It should have at least this feature: when combined in the metalanguage with an appropriate degree-theoretic semantics for the object-language, it should permit one to prove its validity as a logic for the object-language. [23], p130

Although we shall be suggesting that all non-classical theorists adopt a bivalent semantics for the object language, even if their logic can be classically characterised by, say, degree theoretic semantics, we agree that our logic should have the property Williamson suggests. Whatever the logic of the metalanguage, it should permit one to prove its own validity as a logic for the object-language.

Williamson continues:

[This] constraint is not vacuous either, for classical logic clearly fails it. If one combines a classical logic in the metalanguage with a continuum-valued semantics for the object-language, one can prove that classical logic is not valid for the object language. Unfortunately, it is not clear what logics do meet the consraint. One could devise an ad hoc logic to meet it, with a resticted version of the law of excluded middle corresponding to the assumption that all vagueness is first order, but such an assumption has just been seen to be unmotivated. [23], p130

In answer to Williamson's challenge, we shall show for a general class of logics, $L$, how to develop a model theory for the propositional fragment of $L$ in a weak non-standard set theory. The model theory is both faithful, and one can prove in the metatheory, using $L$, the soundness of the model theory for the propositional fragment of $L$.

We begin by outlining a general class of non-classical first order logics, which I name $C$-logics. For each such logic we shall show how to naturally extend it to accommodate second order quantification in a way that ensures that its comprehension schema can be understood open endedly. In other words: we develop a very simple theory of vague sets for that logic. Within the second order theory it is possible to provide a (bivalent) model theory for the propositional calculus that (a) entails, in a certain sense, soundness and completeness of that logic with respect to the model theory (b) validates the logic we are interested in and (c) has the resources to describe the intended model of the propositional calculus.

We shall show that for any $C$-logic, $L$, one can formulate a metatheory $T_{L}$ for the propositional calculus with the following conditions

- One can define formulae $\operatorname{Valid}(x)$ and $\operatorname{Prov}(x)$ that express $L$-validity and $L$-theoremhood of propositional formulae.

- $T_{L}$ proves, in $L, \operatorname{Prov}(\ulcorner\phi\urcorner)$ from $\operatorname{Valid}(\ulcorner\phi\urcorner)$.

- $T_{L}$ proves, in $L, \operatorname{Valid}(\ulcorner\phi\urcorner)$ from $\operatorname{Prov}(\ulcorner\phi\urcorner)$. 
A couple of remarks are in order. It should be noted that, because we are presenting these results for a general class of logics without knowing which principles each has, these results are not constructive - no specific proofs in the metatheory are given. Secondly, the class of logics to which these results apply includes some that have no recursive axiomatic basis. Therefore 'provable' here may not be taken to mean 'provable in some recursively specified axiomatic system', and may sometimes have to be taken to mean 'provable in a nonrecursive system'.

\section{$2.1 \quad C$-logics}

The results that follow apply to a class of second order logics which I shall call $C$-logics. $C$-logics are rich enough to formulate a model theory for their own propositional fragment. In this section we shall define $C$-logics.

Definition 2.0.1. A $C$-algebra is an ordered quadruple $\left\langle\mathcal{V}, \leq,{ }^{*}, \Rightarrow\right\rangle$ where

- $\langle\mathcal{V}, \leq\rangle$ is a complete lattice. i.e.

$-\leq$ is a partial order.

- Every subset of $\mathcal{V}$ has suprema and infima under $\leq$.

- $*$ and $\Rightarrow$ are unary and binary functions respectively on $\mathcal{V}$ to itself.

- $x \Rightarrow y=1$ if and only if $x \leq y$.

For $S$ and $\{x, y\} \subseteq \mathcal{V}$ write $\sqcap S, \sqcup S, x \sqcap y$ and $x \sqcup y$ for the infimum and supremum of $S$ and $\{x, y\}$ respectively. We write 0 and 1 for $\Pi \mathcal{V}$ and $\sqcup \mathcal{V}$.

Notice that the constraints above ensure that for any $x, y$ in a $C$-algebra, $(x \Rightarrow y) \sqcap(y \Rightarrow x)=1$ iff $x=y$. Note also that $*$ (which will eventually function as the interpretation of negation) is completely unconstrained. Say that a $C$-algebra has a reasonable negation just in case $x^{*}=1$ only when $x=0$. This condition holds automatically when $x^{*}$ is defined as $x \Rightarrow 0$.

A $C$-logic is a logic whose consequence relation is characterised by some $C$ algebra, in a way to be defined below. $C$-algebras provide classical algebraic model theory for the logics we are interested in and therefore cannot form the basis of a non-classical metatheory of the kind we have argued for. Among the class of possible logics, some are characterised by $C$-algebras and some are not; the fact that some logics can be characterised by a classical model theory, however, does not mean they can't be given a non-classical model theory as well. The use of classical model theory should be seen only as a way to distinguish logics to which the results in this paper apply from those to which these results do not.

Let's now give a more precise definition of when a logic is 'characterised' by a $C$-algbra. The languages we are concerned with are monadic second order languages with mixed relation symbols. A relation symbol, $P_{i}^{n}$, is mixed when its arguments contain both first order terms and second order terms. I shall use 
$V a r_{1}$ and $V a r_{2}$ to denote the denumberable set of first order and second order variables respectively.

Definition 2.0.2. An assignment over a set $\mathcal{D}$ and a $C$-algebra, $\langle\mathcal{V}, \leq, *, \Rightarrow\rangle$, is a function $v$ such that $v: \operatorname{Var}_{1} \rightarrow \mathcal{D}$ and $v: \operatorname{Var}_{2} \rightarrow \mathcal{V}^{\mathcal{D}}$. Two assignments, $v$ and $u$ are equivalent w.r.t. a variable $x_{i}$, written $v\left[x_{i}\right] u$, iff $v\left(x_{j}\right)=u\left(x_{j}\right)$ for every $j \neq i$.

Definition 2.0.3. A model for a monadic second order language (with mixed relations) is an ordered triple $\langle\mathcal{D},\|\cdot\|, V\rangle . . V$ is a $C$-algebra $\langle\mathcal{V}, \leq, *, \Rightarrow\rangle$. The function $\|\cdot\|_{v}$, with respect to the assignment $v$, over $\mathcal{D}$, obeys the following conditions:

- $\left\|P_{i}^{n}\right\|_{v}: \prod_{k<n} D_{k} \rightarrow \mathcal{V}$ where $D_{k}:=\mathcal{D}$ or $D_{k}:=\mathcal{V}^{\mathcal{D}}$ depending on whether the $k$ th argument of $P_{i}^{n}$ takes a first or second order term.

- $\left\|X_{i}\right\|_{v}=v\left(X_{i}\right)$

- $\left\|x_{i}\right\|_{v}=v\left(x_{i}\right)$

- $\left\|c_{i}\right\|_{v} \in \mathcal{D}$

- $\left\|P_{i}^{n} t_{1}, \ldots, t_{n}\right\|_{v}=\left\|P_{i}^{n}\right\|\left(\left\|t_{1}\right\|_{v}, \ldots,\left\|t_{n}\right\|_{v}\right)$

- $\|\perp\|_{v}=0$

- $\|\phi \wedge \psi\|_{v}=\|\phi\|_{v} \sqcap\|\psi\|_{v}$

- $\|\phi \vee \psi\|_{v}=\|\phi\|_{v} \sqcup\|\psi\|_{v}$

- $\|\phi \rightarrow \psi\|_{v}=\|\phi\|_{v} \Rightarrow\|\psi\|_{v}$

- $\|\neg \phi\|_{v}=\|\phi\|_{v}^{*}$

- $\left\|\forall x_{i} \phi\right\|_{v}=\prod\left\{\|\phi\|_{u} \mid u\left[x_{i}\right] v\right\}$

- $\left\|\exists x_{i} \phi\right\|_{v}=\bigsqcup\left\{\|\phi\|_{u} \mid u\left[x_{i}\right] v\right\}$

- $\left\|\forall X_{i} \phi\right\|_{v}=\prod\left\{\|\phi\|_{u} \mid u\left[X_{i}\right] v\right\}$

- $\left\|\exists X_{i} \phi\right\|_{v}=\bigsqcup\left\{\|\phi\|_{u} \mid u\left[X_{i}\right] v\right\}$

Definition 2.0.4. A formula, $\phi$, is a logical truth with respect to a $C$-algebra, $V$, iff, for every model $\langle\mathcal{D},\|\cdot\|, V\rangle$ and assignment $v$, over $\mathcal{D},\|\phi\|_{v}=1$.

A formula, $\phi$, is a logical consequence of a set of formulae, $\Gamma$, with respect to $V$ iff for every model $\langle\mathcal{D},\|\cdot\|, V\rangle$ and assignment $v$, over $\mathcal{D}$, such that $\|\gamma\|_{v}=1$ for every $\gamma \in \Gamma,\|\phi\|_{v}=1$.

A logic, $L$, is characterised by a $C$-algebra, $V$, iff $L$ 's consequence relation is the same as logical consequence with respect to $V$.

Definition 2.0.5. A logic, $L$, is a $C$-logic if and only if $L$ is characterised by a $C$-algebra. 
When no ambiguity is present, I shall also refer to the propositional or firstorder fragment of a $C$-logic as a $C$-logic as well.

The fuzzy logics commonly used in the study of vagueness form a familiar kind of $C$-logic. The standard fuzzly logics are $C$-logics: basic fuzzy logic, Łukasiewicz logic, Gödel logic and product logic (characterisation theorems can be found in [7].) However, many other familiar logics are $C$-logics: classical logic, intuitionistic logics, BCK and quantum logic to name a few. ${ }^{8}$

It should be noted, however, that these results do not apply to logics lacking a sufficiently strong conditional, such as the strong and weak Kleene logics (which lack the theorem $\phi \rightarrow \phi$ ), and their paraconsistent duals (which lack modus ponens.) Any logic characterised by a $C$-algebra will also validate the inference $\psi \vdash \phi \rightarrow \psi$, therefore logics lacking this rule are excluded as well. ${ }^{9}$ However many logics of interest, including those for reasoning about vagueness and the semantic paradoxes, are included.

Finally, every $C$-logic has the 'rule of proof': either $\Gamma \vdash \phi$ or it's not the case that $\Gamma \vdash \phi .{ }^{10}$ In practice this is not much of a restriction, since rules of proof of this form are had by all non-classical logics that, to my knowledge, have been studied. Even if a logic rejects classical logic generally, it is usually upheld in the proof theory. In the applications involving vagueness and the semantic paradoxes this assumption is justified by the preciseness and nonparadoxicality of the theory of syntax respectively. It is, however, conceivable that an intuitionist might object to this assumption on the grounds that some systems of intuitionistic logic are not decidable. It is up to such an intuitionist to tell us what does count as intuitionistically acceptable reasoning. If this undecidability result is provable in the classically described intuitionistic proof theory, we must reject the classically described proof theory on intuitionistic grounds: the proof theory entails instances of excluded middle it shouldn't. ${ }^{11}$ We therefore have no reason to think that intuitionistically correct reasoning is undecidable. We have at best shown, using incorrect reasoning, that an incorrect system is undecidable. I shall therefore leave this kind intuitionist to one side in what follows.

\subsection{A metatheory for propositional $C$-logics}

In this section we present and axiomatise, for a fixed $C$-logic, $L$, a very simple metatheory for the propositional calculus. The most important aspect of this metatheory is that the logic in which we reason is $L$, and not full classical logic.

The metatheory below is by and large what you would expect to get if you tried to formulate the standard classical model theory for the propositional

\footnotetext{
${ }^{8}$ The conditional in quantum logic is discussed in detail in [8].

${ }^{9}$ The axiom, $\phi \rightarrow(\psi \rightarrow \phi)$, on the other hand fails in any $C$-algebra in which $\mathcal{V}=\left\{0, \frac{1}{2}, 1\right\}$ and in which $a \Rightarrow b=1$ if $a \leq b$ and $a \Rightarrow b=0$ otherwise.

${ }^{10}$ More generally, every $C$-logic has every rule of proof that follows from classical logic. This is a consequence of our defining these logics in classical set theory.

${ }^{11}$ If the undecidability result is only classically provable, then the intuitionist has no reason to accept it anyway.
} 
calculus in the weak set theory we have chosen to reason in. Since we are not working in a full-blown set theory, a few immaterial changes have made. Firstly, instead of treating a model of the propositional calculus as a function from propositional letters to $\{0,1\}$, we have taken a model to be a set of propositional letters. Nonetheless, each such set has a characteristic function, which is a model in the former sense. Secondly, we have taken the relation stating that a sentence is true in a model, $\models$, as a primitive relation symbol and we have axiomatised it. This makes the over all argument easier to follow. In principle, however, the implicit definition of $\models$ could be turned into an explicit one using the second order quantifiers in the usual way.

We shall distinguish sharply between the object language, $\mathcal{L}$ (a propositional language) and the second order metalanguage, $\mathcal{L}^{\prime}$, by using by using $\otimes, \oplus, \sim, \supset$ and $\equiv$ for conjunction, disjunction, negation, implication, and biconditional in $\mathcal{L}$. The metalanguage, $\mathcal{L}^{\prime}$, on the other hand, consists of the following vocabulary

- The logical connectives: $\wedge, \vee, \neg, \rightarrow$ and $\leftrightarrow$

- The logical symbols for first and second order quantification: $\forall, \exists$

- Two non-logical unary predicates: $A(x), \operatorname{Prov}(x)$

- A non-logical binary mixed relation: $X \models y$

- Denumerably many names, $\ulcorner\phi\urcorner$, one for each formula $\phi$ of $\mathcal{L}$

- Denumerably many first and monadic second order variables, $\operatorname{Var}_{1}$ and Var $_{2}$.

Let $L$ be any $C$-logic. We now state, in $L$, the metatheory for the propositional fragment of $L$. Call this $T_{L}$.

1. $A\left(\left\ulcorner p_{n}\right\urcorner\right)$ for each $n \in \mathbb{N}$

2. $\operatorname{Prov}(\ulcorner\phi\urcorner)$ for each theorem and $\operatorname{Prov}(\ulcorner\phi\urcorner) \rightarrow \perp$ for each non-theorem of the propositional fragment of $L$.

3. $X \mid=\left\ulcorner p_{n}\right\urcorner \leftrightarrow X\left\ulcorner p_{n}\right\urcorner$

4. $X \models\ulcorner\phi \oplus \psi\urcorner \leftrightarrow(X \models\ulcorner\phi\urcorner \vee X \models\ulcorner\psi\urcorner)$

5. $X \models\ulcorner\phi \otimes \psi\urcorner \leftrightarrow(X \models\ulcorner\phi\urcorner \wedge X \models\ulcorner\psi\urcorner)$

6. $X \models\ulcorner\sim \phi\urcorner \leftrightarrow \neg(X \models\ulcorner\phi\urcorner)$

7. $X \models\ulcorner\phi \supset \psi\urcorner \leftrightarrow(X \models\ulcorner\phi\urcorner \rightarrow X \mid\ulcorner\psi\urcorner)$

8. $X \models\ulcorner\phi \equiv \psi\urcorner \leftrightarrow(X \models\ulcorner\phi\urcorner \leftrightarrow X \models\ulcorner\psi\urcorner)$

This system allows for simple reasoning about the atomic sentences, provability in $L$, and truth in a model. Axiom 1 ensures that $A$ applies to every atomic 
letter in $\mathcal{L}$. Axiom 2. axiomatises the notion of provability in the propositional fragment of $L$. Here this done by brute force - a thorough metatheorist might wish to set up a classical theory of syntax and provide an axiomatic account of provability. ${ }^{12}$ This approach would only be possible if the logic in question was recursively axiomatisable, and we have made no such assumption here. Fixing the extension of Prov by brute force, as we have done above, allows us more generality. Notice also that we have stated the unprovability of $\phi$ using the conditional instead of negation. If the $C$-logic in question has a reasonable negation, in the sense defined in $\S 2.1$, we may replace ' $\rightarrow \perp$ ' with ' $\neg$ '. Finally the axiom schemata 3 . to 8 . ensure that $\models$ respects the familiar truth clauses for the propositional calculus relative to a model, $X$.

As we have already mentioned, a model in this setting is just a set of atomic sentences. Thus the notion of a model is defined:

$$
\operatorname{Mod}(X):=\forall x(X x \rightarrow A(x))
$$

Presumably there are many interesting metatheoretic definitions and distinctions that can be made in this language, however we shall be particularly interested in the notion of a sentence being valid, or true in all models. This can be stated as follows:

$$
\operatorname{Valid}(\ulcorner\phi\urcorner):=\forall X(\operatorname{Mod}(X) \rightarrow X \models\ulcorner\phi\urcorner)
$$

\subsection{Soundness and completeness}

Say that the model theory described by $T_{L}$ is weakly sound for $L$ iff $\operatorname{Valid}(\ulcorner\phi\urcorner)$ entails (in $\left.T_{L}\right) \operatorname{Prov}(\ulcorner\phi\urcorner)$ ), and say that it is weakly complete for $L$ iff $\operatorname{Prov}(\ulcorner\phi\urcorner)$ entails $\operatorname{Valid}(\ulcorner\phi\urcorner)$. $T_{L}$ proves strong soundness and strong completeness for $L$ just in case $T_{L}$ entails $\operatorname{Prov}(\ulcorner\phi\urcorner) \rightarrow \operatorname{Valid}(\ulcorner\phi\urcorner)$ and $\operatorname{Valid}(\ulcorner\phi\urcorner) \rightarrow \operatorname{Prov}(\ulcorner\phi\urcorner)$ respectively.

In this section we show that the weak soundness and weak completeness of the specified propositional $C$-logic, $L$, holds for the metatheory, $T_{L}$. We shall also show that the metatheory entails the strong soundness theorem.

Since $L$ is a $C$-logic, it's consequence relation is characterised by a $C$-algebra, $V$. It is therefore sufficient to show that every $V$ model of $\operatorname{Valid}(\ulcorner\phi\urcorner)$ is a model of $\operatorname{Prov}(\ulcorner\phi\urcorner)$ and vice versa.

Given a model, $\langle\mathcal{D},\|\cdot\|, V\rangle$, associate formulae of $\mathcal{L}$ with members of $\mathcal{D}$ in the obvious way: for $d \in \mathcal{D}$, associate $d$ with $\phi$ if $\|\ulcorner\phi\urcorner\|=d$. In what follows we shan't distinguish formulae from their associated members of $\mathcal{D}$. Let $v:\|A\| \rightarrow \mathcal{V}$ be a valuation of sentence letters, $\mathcal{S} \mathcal{L}$, in $\mathcal{L}$. Then we define $v^{+}$to be the valuation extended to arbitrary formulae of $\mathcal{L}$ using the algebraic operations as in definition 2.0.3. We first want to show that $\|X=\ulcorner\phi\urcorner\|=\|X\|^{+}(\phi)$.

Proposition 2.1. Suppose $\langle\mathcal{D},\|\cdot\|, V\rangle$ satisfies $T_{L}$ w.r.t $v$. Then $\|X \models\ulcorner\phi\urcorner\|_{v}=$ $\|X\|_{v}^{+}(\phi)$

\footnotetext{
${ }^{12}$ I am assuming that the theory of syntax would be free of kind of phenomena responsible for non-classicality, allowing one to assume classical logic as non-logical principles about syntax. I continue to set aside the intuitionist described in the previous footnote.
} 
Proof. The proof proceeds by induction.

Base case: $\|X \models\ulcorner\phi\urcorner\|_{v}=\|X\ulcorner\phi\urcorner\|_{v}$ by axiom 3. But this $=\|X\|_{v}\left(\|\ulcorner\phi\urcorner\|_{v}\right)=$ $\|X\|_{v}^{+}(\phi)$.

Inductive step: We shall show the case of conjunction by applying axiom 5., the clause for evaluating the semantic value of a conjunction and the inductive hypothesis (in that order) as follows: $\|X \models\ulcorner\phi \otimes \psi\urcorner\|_{v}=\| X \models\ulcorner\phi\urcorner \wedge X \models$ $\ulcorner\psi\urcorner\left\|_{v}=\right\| X \mid\left\ulcorner\ulcorner\urcorner\left\|_{v} \sqcap\right\| X \mid=\ulcorner\psi\urcorner\left\|_{v}=\right\| X\left\|_{v}^{+}(\phi) \sqcap\right\| X\left\|_{v}^{+}(\psi)=\right\| X \|_{v}^{+}(\phi \otimes \psi)\right.$.

The other cases are similar.

Then we get

Proposition 2.2. $\|\operatorname{Valid}(\ulcorner\phi\urcorner)\|=1$ iff for every $f: \mathbb{N} \rightarrow \mathcal{V}, f^{+}(\phi)=1$, where $\|\cdot\|$ is as above.

Proof. $\|\operatorname{Valid}(\ulcorner\phi\urcorner)\|=1$ iff $\|\operatorname{Mod}(X)\|_{v} \Rightarrow\|X \models\ulcorner\phi\urcorner\|=1$ for each $v$ such that $v(X): \mathcal{D} \rightarrow \mathcal{V}$ by the clause for the second order quantifier and the properties of infima. This happens iff $\|\operatorname{Mod}(X)\|_{v} \leq v(X)^{+}(\ulcorner\phi\urcorner)$ for each such $v$ by proposition 2.1 and definition 2.0.2.

Right to left direction: Suppose that for every $f: \mathbb{N} \rightarrow \mathcal{V}, f^{+}(\phi)=1$. In particular this means that $v(X)^{+}(\phi)=1$ for every valuation $v$ so it trivially follows that $\|\operatorname{Mod}(X)\|_{v} \leq v(X)^{+}(\phi)$.

Left to right direction: note that $\|\operatorname{Mod}(X)\|_{v}=1$ if $v(X)(d)>0$ only for $d$ representing propositional letters (i.e. $d$ such that $\|A(x)\|_{x \mapsto d}=1$.) So take any $f: \mathbb{N} \rightarrow \mathcal{V}$ and let $u(X)\left(\left\|\left\ulcorner p_{n}\right\urcorner\right\|\right)=f(n)$ for each $n$ and $u(X)(d)=0$ for the remaining $d$. Clearly, then, $\|\operatorname{Mod}(X)\|_{u}=1$.

If we are supposing that $\|\operatorname{Mod}(X)\|_{v} \leq v(X)^{+}(\ulcorner\phi\urcorner)$ for every valuation $v$ this means that $1 \leq u(X)^{+}(\|\ulcorner\phi\urcorner\|)$ and thus that $f^{+}(\phi)=1$ as required.

Corollary 2.3 (Weak soundness and completeness). Prov $(\ulcorner\phi\urcorner)$ follows in $L$ from $T_{L} \cup\{\operatorname{Valid}(\ulcorner\phi\urcorner)\}$ and $\operatorname{Valid}(\ulcorner\phi\urcorner)$ follows in $L$ from $T_{L} \cup\{\operatorname{Prov}(\ulcorner\phi\urcorner)\}$.

Proof. We'll show that $\|\operatorname{Valid}(\ulcorner\phi\urcorner)\|=1$ iff $\|\operatorname{Prov}(\ulcorner\phi\urcorner)\|=1$ for arbitrary models of $T_{L}$.

By proposition $2.2\|\operatorname{Valid}(\ulcorner\phi\urcorner)\|=1$ iff $\phi$ is true in every propositional model based on $V_{L}$. By definition of $V_{L}$, this happens iff $\phi$ is a theorem of $L$. Iff $\phi$ is a theorem of $L$ then $\operatorname{Prov}(\ulcorner\phi\urcorner)$ is an axiom so $\|\operatorname{Prov}(\ulcorner\phi\urcorner)\|=1$ as required.

Corollary 2.4 (Strong soundness). Prov $(\ulcorner\phi\urcorner) \rightarrow \operatorname{Valid}(\ulcorner\phi\urcorner)$ follows in $L$ from $T_{L}$

Proof. If $\|\operatorname{Prov}(\ulcorner\phi\urcorner)\|=1$ then the result follows from corollary 2.3. If $\|\operatorname{Prov}(\ulcorner\phi\urcorner)\| \neq$ 1 then $\phi$ is not provable in $L$, and so $\|\operatorname{Prov}(\ulcorner\phi\urcorner) \rightarrow \perp\|=1$ by axiom 2 if the metatheory is formulated without a reasonable negation. If the logic has a reasonable negation, then by the $\neg$ version of axiom $2,\|\neg \operatorname{Prov}(\ulcorner\phi\urcorner)\|=1$. In either case $\|\operatorname{Prov}(\ulcorner\phi\urcorner)\|=0$, so the conditional has value 1 . 


\subsection{Failures of strong completeness}

Let us take stock. We have shown that for each $C$-logic, $L$, one can formulate a model theory for the propositional calculus in which the propositional fragment of $L$ is sound according to the model theory. In other words, one can prove the formalisation of the statement 'if $\phi$ is provable, then $\phi$ is true in all models' from the model theory in $L$. One can also prove weak completeness: that the theoremhood of $\phi$ follows (in $L$ ) from our metatheory plus the claim that $\phi$ is true in all models. However we can't in general show the stronger conditional form of completeness

$$
\operatorname{Valid}(\ulcorner\phi\urcorner) \rightarrow \operatorname{Prov}(\ulcorner\phi\urcorner)
$$

which formalises the statement 'if $\phi$ is true in all models, $\phi$ is provable.'

If $L$ admits the deduction theorem, which allows one to move from the fact that $\Gamma \cup\{\phi\}$ entails $\psi$ to the claim that $\Gamma$ entails $\phi \rightarrow \psi,(5)$ would follow from the weak completeness theorem. Thus $C$-logics admitting the deduction theorem, such as intuitionist logic, will automatically be both strongly sound and strongly complete. However, for logics without the deduction theorem strong completeness may fail.

To give a concrete example of such a logic consider Lukasiewicz's continuum valued logic. A calculation shows that the statement ' $((p \rightarrow \neg p) \rightarrow \neg p)$ is true in all models' comes out vague, while it is an entirely sharp matter whether there exists a proof of this formula - there isn't one. In every model of $T_{\mathrm{E}}$, we have $\|\operatorname{Valid}(\ulcorner((p \rightarrow \neg p) \rightarrow \neg p)\urcorner)\|=\frac{1}{2}$ and $\|\operatorname{Prov}((p \rightarrow \neg p) \rightarrow \neg p)\|=0$. So there is instance of strong completeness, namely $\operatorname{Valid}((p \rightarrow \neg p) \rightarrow \neg p) \rightarrow$ $\operatorname{Prov}((p \rightarrow \neg p) \rightarrow \neg p)$, which is vague, since it receives value $\frac{1}{2}\left(=1-\frac{1}{2}+0\right)$ in every model of $T_{\mathrm{E}}$.

(Digression: note that this counterexample only demonstrates that one cannot prove strong completeness in Łukasiewicz logic from the metatheory, $\mathrm{T}_{\mathrm{E}} \cdot{ }^{13}$ However, if we have indeed demonstrated that the notion of provability and the notion of logical consequence are distinct, the fact that strong completeness is not provable from the metatheory does not mean that it is not a consequence of it. The following possibility is still open: that the strong completeness theorem is definitely not provable from the metatheory, even though it is not definitely not a logical consequence of it. Whether or not strong completeness is validly entailed by the metatheory may be a vague matter in the same way that the validity of $((p \rightarrow \neg p) \rightarrow \neg p)$ is vague. $)$

In the metatheory for Eukasiewicz logic, the principle $\operatorname{Valid}(\ulcorner\phi\urcorner) \vee \neg \operatorname{Valid}(\ulcorner\phi\urcorner)$ does not hold unrestrictedly. On the other hand, the notion of provability is precise, which is manifested by the principle $\operatorname{Prov}(\ulcorner\phi\urcorner) \vee \neg \operatorname{Prov}(\ulcorner\phi\urcorner)$. This property is perhaps an advantage of this account of validity. In at least two applications one might expect the source of non-classicality to infect the notion

\footnotetext{
${ }^{13}$ The fact that this notion of 'provability' is most likely not recursively axiomatisable is not the issue here. I assume that 'provable' just means 'provable by some logically omniscient person', much as in the classical case we still count classical theorems as provable even if they are so long and complicated no human could carry out the proof.
} 
of validity but not the notion of provability in $L$. On the one hand, considerations of higher order vagueness lead us to think that the very notions we used to define validity are vague, but the same cannot be said of the notion of provability. On the other hand, considerations very similar to those responsible for the semantic paradoxes can lead one to think that there are similar paradoxes for a validity predicate (see [16]), or a consequence relation (see [1], [22].) Since in this case we should expect classical laws involving the validity predicate and consequence relation, to fail, we shouldn't expect to be able to give it a sound and complete axiomatisation - at least, not if we are using a classical theory of syntax.

In both cases we have reason to think that validity is a vague or indeterminate notion, while provability is not. In these cases we should not expect both strong soundness and completeness to hold, since no vague or indeterminate notion can be determinately equivalent to a determinate one. ${ }^{14}$

\subsubsection{Conditional assertions}

Although I have argued that the failures of strong completeness are desirable for some applications, the problem with the notion of weak completeness is that it is not stateable in the metatheory as it stands. We said that $T_{L}$ provides a weakly complete model theory for $L$ when $\operatorname{Valid}(\ulcorner\phi\urcorner)$ entails in $T_{L} \operatorname{Prov}(\ulcorner\phi\urcorner)$, and this is metametatheoretic statement - a statement about what follows from what in the metatheory. In this section and the next I shall briefly consider two ways of stating weakened versions of soundness and completeness for Łukasiewicz's continuum valued logic in the metatheory.

In [2] Field argues, among other things, that non-classical logicians should make a distinction between the assertion of a conditional and the corresponding conditional assertion. Since paracomplete theorists typically reject the deduction theorem, (a) $\phi \vdash \psi$ and (b) $\vdash(\phi \rightarrow \psi)$ come apart. Field identifies assertions about valid inferences, such as (a), with the validity of the conditional assertion of $\psi$ on $\phi$, whereas (b) is to be associated with the validity of the ordinary assertion of the conditional $(\phi \rightarrow \psi)$.

Think of making the conditional assertion of $\psi$ on $\phi$ as being equivalent to making the ordinary assertion that $\psi$, if $\phi$ obtains, and to making a vacuous assertion otherwise. Making sense of this distinction on the instrumental continuum valued semantics is simple. The conditional assertion of $\psi$ on $\phi$ gets the value of $\psi$ if $\phi$ gets value 1 , and otherwise gets value 1 . The conditional assertion of $\psi$ on $\phi$ is vacuously true if $\phi$ fails to be anything less than fully true. It is important to note, however, that conditional assertion is not like some connective in the language. In particular, it is not embeddable as the ordinary conditional is. What sense can be made of the conjunction of a sentence with a conditional assertion, or any kind assertion for that matter? Speech acts just aren't the kinds of things we can conjoin with sentences.

\footnotetext{
${ }^{14}$ In other cases, such as intuitionistic logic, the notion of provability may fail to be classical when the when the proof theory is not decidable. For the intuitionist who buys the results of section 2 , at any rate, strong completeness is immediate from 2.3 by the deduction theorem.
} 
The distinction is idle unless the antecedent is vague. For the purposes of stating strong completeness, however, we are in exactly such a situation, and, indeed, it is a straightforward consequence of Corollary 2.3 that the conditional assertion of $\operatorname{Prov}(\ulcorner\phi\urcorner)$ on $\operatorname{Valid}(\ulcorner\phi\urcorner)$ receives value 1 on every model of $T$. (The converse conditional assertion, as well as assertion of the conditional $(\operatorname{Prov}(\ulcorner\phi\urcorner) \rightarrow \operatorname{Valid}(\ulcorner\phi\urcorner))$ are also valid.) Thus a version of soundness and completeness can be given by a schema of conditional assertions. If we want a statement of soundness and completeness that has the more familiar biconditional form we'll have to move to a more expressive language.

\subsubsection{Degrees of provability}

Rational Pavelka logic (RPL) is the the addition to Eukasiewicz logic of all the rational truth constants $\bar{\gamma}$ for $\gamma \in[0,1] \cap \mathbb{Q}$, and corresponding axioms (see Hajek [7].) In this extended language there is a natural notion of the 'degree of provability' of $\phi$, namely, $\sup \left\{\gamma \vdash_{R P L}(\bar{\gamma} \rightarrow \phi)\right\}$ - write this as $|\phi|$. Letting $\langle\phi\rangle:=\inf \{\|\phi\| \mid\|\cdot\|$ a model $\}$ there a version of completeness for RPL:

$$
|\phi|=\langle\phi\rangle
$$

Let $\gamma_{\phi}:=|\phi|$. It is easy to verify that $\|\operatorname{Valid}(\ulcorner\phi\urcorner)\|=\langle\phi\rangle$ for every second order model $\langle\mathcal{D},\|\cdot\|, V\rangle$ of $T_{L}$. We are then in a position to state that soundness and completeness, given by the following schema, is a logical consequence of $T_{L}$ :

$$
T \models \operatorname{Valid}(\ulcorner\phi\urcorner) \leftrightarrow \bar{\gamma}_{\phi}
$$

One worry you might have with this method, is that it seems to be parasitic on the continuum valued model theory. The continuum valued model theory was supposed to be nothing more than a ladder, a neat way to understand the consequence relation, which, ultimately, was to be thrown away once climbed. On this approach it seems to serve a more important role - we cannot throw away the ladder, since we permanently have the constants $\bar{\gamma}, \gamma \in[0,1] \cap \mathbb{Q}$ in our language. Our understanding of these constants seems to be completely dependent on our understanding of the continuum valued semantics.

Although I am not yet entirely sure whether we can understand the $\bar{\gamma}$ without the continuum valued semantics, I conjecture that one only needs to add $\overline{\frac{1}{2}}$ to Lukasiewicz logic to have all the necessary instances of (7). $\frac{\overline{1}}{2}$ 's meaning is completely determined by it's axiomatisation:

- $\left((p \wedge \neg p) \rightarrow \frac{\overline{1}}{2}\right)$

$$
\text { - }\left(\frac{\overline{1}}{2} \rightarrow(p \vee \neg p)\right)
$$

Since these axioms are all one needs to understand $\frac{1}{2}$, its use is not parasitic on the continuum valued semantics. ${ }^{15}$ We can think of it as a statement which is determinately vague, i.e. vague but not higher order vague.

\footnotetext{
${ }^{15}$ Indeed, it is already definable in second order $\mathrm{\iota}: \exists x \forall X(X x \vee \neg X x)$.
} 


\subsection{Final remarks}

We have shown that one can carry out classical model theory in a non-classical setting. What of classically described non-bivalent model theory? To continue with our pet example: what about the continuum valued semantics for $€$ ? The results presented here can be used to give a Kreisel-style argument that the classical metatheory of continuum valued semantics gives an extensionally adequate characterisation of logical consequence understood as truth in all possible bivalent interpretations (modulo the issues to do with the deduction theorem and strong completeness.) The extension of $\operatorname{Prov}(\cdot)$ in $T_{\mathrm{E}}$ was simply stipulated to be truth on every classically described continuum valued model, or in the general case, truth on every $\mathcal{V}$ valued model, and $\operatorname{Valid}(\cdot)$ was designed to express the notion of truth on every non-classically described bivalent model: the results of $\S 2.1$ demonstrate that these two notions coincide.

This argument assumes that bivalent models play the same role with respect to the continuum valued models as the class models do to the set sized models in Kreisel's argument. In other words, that they are the intensionally correct way to characterise consequence. What of the view that takes the continuum valued degree semantics seriously, and does not recognise bivalent consequence as the antecedent notion of logical consequence. This is perhaps the clearest target in Williamsons' criticism in the passage cited. Williamson points out that this view seems to be unstable, for according to the degree semantics conditional excluded middle, $(p \rightarrow q) \vee \neg(p \rightarrow q)$, comes out as valid (since truth values are linearly ordered) while the more general law of excluded middle does not. ${ }^{16}$ But, he argues, it is the general law of excluded middle, not conditional excluded middle, that is required in the metalanguage to show that $(p \rightarrow q) \vee \neg(p \rightarrow q)$ is valid. The natural argument for the validity of CEM begins 'either the truth value of $p$ is less than or equal to the truth value of $q$ or it isn't' and this is an instance of excluded middle and not conditional excluded middle.

This objection, however, is not as clear cut as it might at first seem. One might just take this to mean that conditional excluded middle doesn't hold on this degree semantics after all, provided you consider all truth assignments, including vague ones. So truth values, despite appearances, aren't linearly ordered. (Certainly $[0,1]$ is linearly ordered, but it doesn't follow that all functions, $v$, into $[0,1]$, including vague functions, are linearly ordered in the sense that either $v(p) \leq v(q)$ or $v(q) \leq v(p)$ for any $p$ and $q .{ }^{17}$ ) Alternatively one could add further constraints on acceptable truth value assignments that would be vacuous in a classical setting. For example we could stipulate that we only consider truth value assignments, $v$, to $[0,1]$ in which $v(p) \leq v(q)$ or $v(p) \not \leq v(q)$ for any $p$ and $q$. This does not entail there is no higher order vagueness as there can still be failures of excluded middle elsewhere in the metalanguage. For a

\footnotetext{
${ }^{16}$ Actually Williamson is considering a slightly different, intuitionistic, continuum valued semantics in which CEM comes out true. Essentially the same point can be made for Lukasiewicz logic for the law $(p \rightarrow q) \vee(q \rightarrow p)$.

${ }^{17}$ This is demonstrated by the function $v$ on the linearly ordered set $\{0,1\}$ constructed in $\S 1$.
} 
contrived example take the function $v$ defined in $\S 1$ as mapping $p_{i}$ to 1 if $i$ is small and to 0 otherwise. Then it's determinate that $v\left(p_{i}\right) \leq v\left(p_{j}\right)$ whenever $j \leq i$, which stated more carefully says $\forall x y\left(\left(\left\langle p_{i}, x\right\rangle \in v \wedge\left\langle p_{j}, y\right\rangle \in v\right) \rightarrow x \leq y\right)$, and thus $v\left(p_{i}\right) \leq v\left(p_{j}\right) \vee v\left(p_{i}\right) \not \leq v\left(p_{j}\right)$ holds for any $i$ and $j$, but claims like $v\left(p_{i}\right)=1 \vee v\left(p_{i}\right) \neq 1$ are not in general true.

The philosophical issues involved in combining non-classical semantics with non-classical logic are complex and clearly are not done justice by the few remarks I make here. I shall have to leave the full investigation of these matters to a future project. However, I take it I have shown there is a clear sense in which non-classical logicians can carry out important metatheoretic reasoning in their own logic in a bivalent model theory.

\section{References}

[1] J. Beall and J. Murzi. Two flavors of curry paradox. To appear.

[2] H. Field. Saving truth from paradox. Oxford University Press.

[3] H. Field. Indeterminacy, degree of belief, and excluded middle. Nous, $34(1): 1-30,2000$.

[4] H. Field. Solving the paradoxes, escaping revenge. Revenge of the liar: new essays on the paradox, page 78, 2007.

[5] K. Fine. The permutation principle in quantificational logic. Journal of Philosophical Logic, 12(1):33-37, 1983.

[6] VN Grišin. Predicate and set-theoretic calculi based on logic without contractions. Mathematics of the USSR-Izvestiya, 18:41, 1982.

[7] P. Hájek. Metamathematics of fuzzy logic. Kluwer academic publishers, 2001.

[8] G.M. Hardegree. The conditional in quantum logic. Synthese, 29(1):63-80, 1974.

[9] G. Kreisel. On weak completeness of intuitionistic predicate logic. Journal of Symbolic logic, pages 139-158, 1962.

[10] G. Kreisel. Informal rigour and completeness proofs. Studies in Logic and the Foundations of Mathematics, 47:138-186, 1967.

[11] H. Leitgeb. On the Metatheory of Field's 'Solving the Paradoxes, Escaping Revenge'. Revenge of the liar: new essays on the paradox, page 159, 2007.

[12] D.C. McCarty. Incompleteness in intuitionistic metamathematics. Notre Dame journal of formal logic, 32(3):323-358, 1991. 
[13] D.C. McCarty. On Theorems of Gödel and Kreisel: Completeness and Markov's Principle. Notre Dame Journal of Formal Logic, 35(1):99-107, 1994.

[14] V. McGee. How we learn mathematical language. The Philosophical Review, 106(1):35-68, 1997.

[15] V. McGee. Review: John etchemendy, the concept of logical consequence. Bulletin of Symbolic logic, 7(3):379-380, 2001.

[16] R. Montague. Syntactical treatments of modality, with corollaries on reflexion principles and finite axiomatizability. Acta philosophica fennica, 16:153-167, 1963.

[17] A. Rayo and G. Uzquiano. Toward a theory of second-order consequence. Notre Dame Journal of Formal Logic, 40(3):315-325, 1999.

[18] A. Rayo and T. Williamson. A completeness theorem for unrestricted firstorder languages. Liars and Heaps: New Essays on Paradox, pages 331-56, 2004.

[19] A. Tarski. On the concept of logical consequence. Philosophy of logic: an anthology, 2002.

[20] M. Tye. Sorites Paradoxes and the Semantics of Vagueness. Philosophical Perspectives, 8:189-206, 1994.

[21] P. Welch. Ultimate Truth vis-a-vis Stable Truth. The Review of Symbolic Logic, 1(01):126-142, 2008.

[22] B. Whittle. Dialetheism, logical consequence and hierarchy. Analysis, 64(284):318-326, 2004.

[23] T. Williamson. Vagueness. Routledge, 1994. 